\title{
A TENSOR APPROACH TO INTERPOLATION PHENOMENA IN DISCRETE ABELIAN GROUPS
}

\author{
RON C. BLEI
}

ABSTRACT. We show that a tensor algebra setting is an efficient tool that separates Sidonicity from other interpolation properties.

In this note we show that a tensor algebra setting [11] is a convenient framework where Sidonicity can be efficiently separated from other interpolation properties. Non-Sidon $\Lambda(p)$-sets in any $\Gamma$ (see $A$ below) were first obtained in [3] and [5]; our proof, similar to the one in [3, Théorème 5, p. $359]$ is more direct. The Rosenthal property (B) of some non-Sidon sets was first displayed in [9], where an appeal was made to the notion of "sup-norm partitions" (see also [1] and [2]). C was first observed in [4].

In what follows below, $\Gamma$ is a discrete abelian group, and $\hat{\Gamma}=G$. Without loss of generality, we assume that $\Gamma$ is a countable group. We refer to [11] for standard notation and facts. Let $E \subset \Gamma$; we set

and

$$
A(E)=L^{1}(G) \hat{\wedge} /\left\{f \in L^{1}(G): \hat{f}=0 \text { on } E\right\},
$$

$$
B(E)=M(G) \hat{\gamma} /\{\mu \in M(G): \hat{\mu}=0 \text { on } E\} .
$$

If $K(G)$ is a subspace of $L^{1}(G)$, we set

$$
K_{E}(G)=\{f \in K(G): \hat{f}=0 \text { off } E\} .
$$

Definitions. (a) $E \subset \Gamma$ is a Sidon set if $L_{E}^{\infty}(G)=A_{E}(G)\left(=l^{1}(E) \hat{)}\right.$; equivalently, $A(E)=c_{0}(E)$.

(b) Let $1<p<\infty$. $E \subset \Gamma$ is a $\Lambda(p)$-set if $L_{E}^{1}(G)=L_{E}^{p}(G)$.

(c) $E$ is a Rosenthal set if $L_{E}^{\infty}(G)=C_{E}(G)$.

(d) Let $1 \leq p<2$. $E$ is a $p$-Sidon set if $C_{E}(G)^{\wedge} \subset l^{p}(E)$.

If $E$ is a Sidon set, then $E$ is a Rosenthal set, a $p$-Sidon set, and a $\Lambda(p)$-set, for all $p$. The first two claims are trivial to verify, whereas the third is not (see 5.7.7 of [11], and A below).

Received by the editors March 18, 1974.

AMS (MOS) subject classifications (1970). Primary 43A25.

Key words and phrases. Sidon set, $\Lambda(p)$-set, $p$-Sidon set. 
Definition. We say that $S \subset \Gamma$ is a dissociate set if whenever the equality $\Sigma_{j=1}^{N} \omega_{j} \alpha_{j}=0$, where $\omega_{j}=-1,0,1$, and $\left\{\alpha_{j}\right\}_{j=1}^{N} \subset S$, implies that $\omega_{j}=0$ for all $j$.

It is well known that every infinite discrete abelian group contains a dissociate set.

Definition. Let $S_{1}$ and $S_{2}$ be any countably infinite sets. We set

$$
\begin{array}{r}
l^{\infty}\left(s_{1}\right) \hat{\otimes} l^{\infty}\left(s_{2}\right)=\left\{\phi \in l^{\infty}\left(s_{1} \times S_{2}\right): \phi=\sum f_{j} g_{j} \text {, where } f_{j} \in l^{\infty}\left(s_{1}\right),\right. \\
\left.g_{j} \in l^{\infty}\left(s_{2}\right), \text { and } \sum\left\|f_{j}\right\|_{\infty}\left\|g_{j}\right\|_{\infty}<\infty\right\} .
\end{array}
$$

We define $c_{0}\left(S_{1}\right) \hat{\otimes} c_{0}\left(S_{2}\right)$ similarly, and for $\phi \in l^{\infty}\left(S_{1} \times S_{2}\right)$ or $c_{0}\left(S_{1} \times S_{2}\right)$ we set $\|\phi\|_{\otimes}=\inf \left\{\Sigma\left\|f_{j}\right\|_{\infty}\left\|g_{j}\right\|_{\infty}: \phi=\Sigma f_{j} g_{j}\right\}$. We refer to [12] for a study of the above tensor algebras. We shall need the following:

Fact $V$ (Theorem 3.1 of [11]). Let $E$ and $F$ be infinite disjoint subsets of $\Gamma$ so that $E \cup F$ is dissociate. Then $A(E+F) \approx c_{0}(E) \hat{\otimes} c_{0}(F)$, and $l^{\infty}(E) \hat{\otimes} l^{\infty}(F)$ is a closed subalgebra of $B(E+F)$. (Note that we can freely associate functions in $l^{\infty}(E+F)$ with functions in $l^{\infty}(E \times F)$.)

By the above, since $c_{0}(E) \hat{\otimes} c_{0}(F) \varsubsetneqq c_{0}(E+F)$ (see, for example, Lemma VIII.10.5 of [6]), $E+F$ is not a Sidon set.

In what follows below, $E=\left\{\lambda_{j}\right\}_{j=1}^{\infty}$ and $F=\left\{\nu_{j}\right\}_{j=1}^{\infty}$ are infinite disjoint subsets of $\Gamma$, and $E \cup F$ is a dissociate set.

A. $E+F(E+E)$ is a $\Lambda(p)$-set for all $p$. The proof that every Sidon set in $\Gamma$ is a $\Lambda(p$ )-set for all $p$ (see 5.7.7 in [11]) is based on the prior knowledge that an infinite independent set in $\bigoplus z_{2}$ is a $\Lambda(p)$-set for all $p$. We follow a similar route by making use of

Lemma. There exists $\Gamma_{1}$, an infinite discrete abelian group $\left(\hat{\Gamma}_{1}=H\right)$, and $S_{1}=\left\{\alpha_{j}\right\}_{j=1}^{\infty}, S_{2}=\left\{\beta_{j}\right\}_{j=1}^{\infty}$, two infinite disjoint subsets of $\Gamma_{1}$, so that $S_{1} \cup S_{2}$ is dissociate and $S_{1}+S_{2}$ is a $\Lambda(p)$-set for all $p$.

See [9], for example.

Now, let $p>2$ and let $f$ be any trigonometric polynomial in $L_{E+F}^{1}(G)$ :

$$
f(g)=\sum a_{i j}\left(\lambda_{i}+\nu_{j}, g\right) .
$$

We use $S_{1}$ and $S_{2}$ in $\Gamma_{1}$ of the above Lemma to perturb $\hat{f}$ :

$$
f_{h}(g)=\sum a_{i j}\left(a_{i}, h\right)\left(\beta_{j}, h\right)\left(\lambda_{i}+\nu_{j}, g\right) \quad \text { for all } h \in H .
$$

We now note that for each $h \in H$, there exists $\mu=\mu_{b}$ in $M(G)$ so that 


$$
\hat{\mu}_{h}\left(\lambda_{j}+\nu_{i}\right)=\overline{\left(\alpha_{i}, h\right)\left(\beta_{j}, h\right)}
$$

and $\sup _{b}\left\|\mu_{b}\right\|_{M}=b<\infty$. This follows immediately from Fact V (see Remark (i)). We proceed exactly as in 5.7.7 of [11]: $f=f_{b} * \mu_{h}$, and therefore

$$
\|f\|_{p} \leq\left\|f_{h}\right\|_{p}\left\|\mu_{h}\right\|_{M} \leq b\left\|f_{h}\right\|_{p} \text {. }
$$

That is,

$$
\int_{G}|f(g)|^{p} d g \leq b^{p} \int_{G}\left|\sum a_{i j}\left(a_{i}, h\right)\left(\beta_{j}, h\right)\left(\lambda_{i}+\nu_{j}, g\right)\right|^{p} d g .
$$

We integrate both sides over $H$, interchange the order of integration, and apply the above Lemma.

Remarks. (i) We do not have to appeal to Fact V: We can obtain (*) directly by considering the Riesz product whose transform equals $\overline{\left(\alpha_{i}, b\right)}$ at $\lambda_{i}$, and $\overline{\left(\beta_{j}, h\right)}$ at $\nu_{j}$

(ii) The $\Lambda(p)$ constants of $E+F$ are inherited from $S_{1}+S_{2}$ in $\Gamma_{1}$. In fact, by interchanging the roles of $G$ and $H$ in the above proof, we see that the behavior of the $\Lambda(p)$ constants of $E+F$ is the same in all groups.

(iii) The above argument can be easily modified to prove that $E+E$ is a $\Lambda(p)$-set for all $p$.

B. $E+F$ is a Rosenthal set (see also [8, p. 251]). We note that (1)

$$
\left(c_{0}(E) \hat{\otimes} c_{0}(F)\right)^{*}=\left\{\left(a_{m n}\right): \exists C>0 \text { so that }\left|\sum a_{m n} x_{m n} y_{n}\right| \leq C\|x\|_{\infty}\|y\|_{\infty}\right.
$$

$$
\text { for all } \left.x, y \in c_{0}(E), c_{0}(F) \text { respectively }\right\} \text {. }
$$

But, $\left(c_{0}(E) \hat{\otimes} c_{0}(F)\right)^{*}=L_{E+F}^{\infty}(G)^{\hat{n}}$, and since the right-hand side of (1) can be viewed as a sup-norm closure of trigonometric polynomials with spectrum in $E+F$, our assertion follows.

C. $E+F$ is a 4/3-Sidon set. By (1) above, the assertion is precisely the content of the third inequality in Theorem 1 (1) of Littlewood [7]:

$$
\left(\sum \sum\left|a_{m n}\right|^{4 / 3}\right)^{3 / 4} \leq A\left(\sup _{x, y ;\|x\|_{\infty},\|y\|_{\infty} \leq 1}\left|\sum a_{m n} x_{m} y_{n}\right|\right) \text {. }
$$

Littlewood observes in [7] that the $4 / 3$ is sharp.

\section{REFERENCES}

1. R. C. Blei, On trigonometric series associated with separable, translation invariant subspaces of $L^{\infty}(G)$, Trans. Amer. Math. Soc. 173 (1972), 491-499.

2. - A simple diophantine condition in harmonic analysis, Studia Math. 52 (1974), 195-202. 
3. A. Bonami, Étude des coefficients de Fourier des fonctions de $L^{p}(G)$, Ann. Inst. Fourier (Grenoble) 20 (1970), fasc. 2, 335-402 (1971). MR 44 \#727.

4. R. E. Edwards, and K. A. Ross, p-Sidon sets, J. Functional Analysis 15 (1974), 404-427.

5. R. E. Edwards, E. Hewitt and K. A. Ross, Lacunarity for compact groups. I, Indiana Univ. Math. J. 21 (1971/72), 787-806. MR 45 \#6981.

6. Y. Katznelson, An introduction to harmonic analysis, Wiley, New York, 1968. MR 40 \#1734.

7. J. E. Little wood, Quart. J. Math. 1 (1930), 164-174.

8. Y. Meyer, Recent advances in spectral synthesis, Conf. on Harmonic Analysis, College Park, Maryland, 1971, Lecture Notes in Math., Springer-Verlag.

9. H. P. Rosenthal, On trigonometric series associated with weak* closed subspaces of continuous functions, J. Math. Mech. 17 (1967), 485-490. MR 35 \#7064.

10. W. Rudin, Trigonometric series with gaps, J. Math. Mech. 9 (1960), 203227. MR $22 \# 6972$.

11. - Fourier analysis on groups, Interscience Tracts in Pure and Appl. Math., no. 12, Interscience, New York, 1962. MR 27 \#2808.

12. N. Varopoulos, Tensor algebras over discrete spaces, J. Functional Analysis 3 (1969), 321-335. MR $40 \# 3328$.

DEPARTMENT OF MATHEMATICS, UNIVERSITY OF CONNECTICUT, STORRS, CONNECTICUT 06268 